\section{The Los Angeles Healthy Community Neighborhood Initiative: A Ten Year Experience in Building and Sustaining a Successful Community-Academic Partnership}

\author{
Keyonna M King', D’Ann Morris'2, Loretta Jones ${ }^{3}$, Aziza \\ Lucas-Wright ${ }^{3}$, Felica Jones ${ }^{3}$, Homero E del Pino ${ }^{1,4}$, \\ Courtney Porter ${ }^{2,4}$, Roberto Vargas ${ }^{1,4}$, Katherine Kahn', \\ Arleen F Brown ${ }^{1}$, Keith C Norris ${ }^{1 *}$ and for the HCNI Study \\ Group
}

${ }^{1}$ Division of General Internal Medicine and Health Services Research, University of California, Los Angeles

${ }^{2}$ Division of General Internal Medicine and Health Services Research, Los Angeles Urban League, University of California, Los Angeles, California, USA

${ }^{3}$ Healthy African American Families, University of California, Los Angeles, California, USA

${ }^{4}$ Department of Aging, Charles R Drew University, Los Angeles, California, USA

\begin{abstract}
Background: Developing effective Community-Academic Partnerships (CAPs) is challenging, and the steps to build and sustain them have not been well documented. This paper describes efforts to form and sustain the Healthy Community Neighborhood Initiative (HCNI), a CAP to improve health in a low-income community in South Los Angeles.

Methods: Moderated, semi-structured discussions with HCNI community and academic partners were used to develop a framework for CAP formation.

Results: We identified two key features, shared values and respect, as critical to the decision to form the HCNI. Five elements were identified as necessary for building and sustaining the $\mathrm{HCNI}$ :

*Corresponding author: Keith C Norris, Division of General Internal Medicine and Health Services Research, University of California, 911 Broxton Plaza, Room 103, Los Angeles, CA, USA, Tel: +1 3107948650; Fax: +1 3107940724 ; E-mail: kcnorris@mednet.ucla.edu

Citation: King KM, Morris D, Jones L, Lucas-Wright A, Jones F, et al. (2015) The Los Angeles Healthy Community Neighborhood Initiative: A Ten Year Experience in Building and Sustaining a Successful Community-Academic Partnership. J Community Med Public Health Care 2: 007.
\end{abstract}

Received: March 24, 2015; Accepted: May 30, 2015; Published: June 15, 2015 trust, transparency, equity and fairness, adequate resources and developing protocols to provide structure. We also identified several challenges and barriers and the strategies used in the HCNI to mitigate these challenges.

Conclusion: We developed a framework to incorporate and reinforce the key elements identified as crucial in building and sustaining a CAP in a low-income community.

Keywords: Building partnerships; Community-academic partnership; Community-based participatory research; Memorandum of understanding; Sustaining partnerships

\section{Introduction}

A growing interest in community engaged research approaches to establish and sustain healthier communities and improve population health [1-4] has produced an increasing number of research studies that involve key community stakeholders in all aspects of the research process: from the identification of health priorities to study design, implementation, data analysis and dissemination [5]. Community engaged research may occur at a variety of levels of intensity ranging from the use of Community Advisory Boards (CABs) to fully engaged Community-Based Participatory Research (CBPR). Israel and colleagues [5] have defined CBPR as "a collaborative approach to research that equitably involves all partners in the research process and recognizes the unique strengths that each brings, to jointly explore answers to complex socio-medical questions." We also frequently use of the term "Community-Partnered Participatory Research" (CPPR) to emphasize partnering with communities rather than merely possessing a location within the community to conduct research [6-8]. The idea of community and academia partnering in the research process leads to the need for a better understanding how to build and sustain such partnerships. There are several examples in the literature of "how to" build a Community-Academic Partnership (CAP) [9-17], but developing effective CAPs is challenging [18], and the steps to build and sustain these CAPs have not been well documented. Most reports of CAPs have instead focused on discussing accomplishments [19-22], while a few studies have reported on selected elements of successful collaborations, such as building trust and team-building activities $[23,24]$. There is a clear need for a better understanding of possible effective strategies [1].

The HCNI is a pilot project started in 2005 consisting of community-academic partnerships between 2 community organizations and 2 academic institutions: Los Angeles Urban League (LAUL), Healthy African American Families Phase II (HAAF), Charles R Drew University (CDU), and University of California Los Angeles (UCLA). The overall goal of HCNI is to identify and address health-related questions posed by the residents in Park Mesa Heights, a low-income community in South Los Angeles. The initiative initially focused on a 70-square block area documented to have some of the poorest health outcomes for preventable chronic disease and some of the highest rates of premature morbidity and mortality among African Americans and Latinos in Los Angeles County [25]. 


\section{HCNI: 70-square block area}

In 2005, the LAUL and United Way of Greater Los Angeles produced "The State of Black Los Angeles," a report that measured the equality index, an objective tool to assess overall well-being of racial/ethnic minority groups in Los Angeles County (LAC) in six domains: economics, housing, health, education, criminal justice, and civic engagement [25]. The report indicated that African Americans in LAC fair worse than any other racial/ethnic group in all areas except civic engagement. The results presented in the report prompted community organizations to actively address these concerns. The HCNI partners recognized to improve the health of the community, each element of the report needed to be addressed simultaneously.

The residents of the 70 -square block area are disproportionately affected by hypertension, obesity, diabetes, hyperlipidemia, and heart disease and face many barriers to implementing health promotion strategies and accessing health care for these conditions. The problem is magnified because addressing these health problems requires more than a focus on health care. Most health outcome interventions do not address any social determinants of health and those that do, typically focus on one domain independent of the others [25].

This paper uses in-depth qualitative analyses to describe the formation and evolution of the CAP for the HCNI, a project designed to address health disparities in this community in South Los Angeles. We provide lessons learned in building a sustainable CAP and offer insights into strategies for stakeholders interested in forming and nurturing effective CAPs.

\section{Methods}

Our team used CBPR/CPPR methods to build a foundation for the Healthy Community Neighborhood Initiative (HCNI), a CAP to improve the health of residents of a low income, bi-ethnic neighborhood in South Los Angeles (LA). Our project has been approved by both Charles R Drew University and University of California, Los Angeles Institutional Review Boards. The data presented in this paper did not require additional IRB approval since it was part of the internal evaluation of the project.

\section{Description of partners}

The LA Urban League (LAUL) is a non-profit community organization founded in 1921 focused on advancing equal opportunities on behalf of African Americans, Latinos and other minority youth and adults. Its mission is to enable these groups to secure economic self-reliance, parity, power and civil rights and a high quality of life through advocacy. LAUL employees have expertise in many of the social determinants of health, including education, employment, housing and criminal justice.

Healthy African American Families (HAAF) Phase II is a non-profit community serving organization founded in 1992. They facilitate health research and health promotion activities through education, training, and collaboration. HAAF has over 25 years of history partnering locally and nationally with community, academia, researchers, and government [26].

Charles R Drew University (CDU) is located in the diverse and underserved area of South Los Angeles. CDU was chartered in 1966 as a post-graduate school in response to the Watts rebellion, and the medical education program was established in 1978 as a satellite of the UCLA School of Medicine. The CDU mission is to provide education, research, and clinical service in the context of community engagement.

The University of California, Los Angeles Clinical and Translational Science Institute (UCLA CTSI) is comprised of four partner institutions-Cedar-Sinai Medical Center, CDU, LA Biomed/Harbor-UCLA, UCLA-Westwood-and a network of community partners. The CTSI's central mission is to translate evidence-based research into effective clinical practices, public health initiatives and policies that address the greatest health needs of LA County.

\section{Formation of the partnership}

Based on the findings from "The State of Black Los Angeles," the LAUL's Deputy Neighborhood Officer for Health (DM) initiated a series of neighborhood discussions on health with key community stakeholders, including HAAF (LJ), and academic partners at CDU $(\mathrm{KN})$. During initial meetings to establish the partnership, each partner discussed his or her organization's or institution's goals and the team worked to develop a shared vision and set of goals for the project and for building the partnership. This core group of leaders was subsequently joined by other members of the UCLA CTSI. Once the team established a shared vision, the partnership became more defined. Leadership meetings included all partners and were held weekly to review study design, develop protocols and surveys, analyze results, and review draft products (e.g., scientific abstracts, community reports, presentations and manuscripts). The team also held quarterly community LAUL Health Collaborative stakeholder/advisory meetings to provide feedback and get further input. Additional weekly meetings and/or calls with coauthors were held for manuscript preparation. Meetings were held at HAAF or LAUL offices, or occasionally other local community sites. To date, the HCNI study team consists of approximately 17 members, not including undergraduate, graduate and professional student interns.

The partnership was uniquely positioned to launch the HCNI for numerous reasons. Several of the community and academic partners worked together on community research and outreach projects for as many as 10 to 15 years prior to the formation of the HCNI, which established a foundation of respect, trust and familiarity. This decreased the time required to build trust and familiarity in a CAP. Moreover, the community partners have spearheaded or collaborated on nationally recognized work on the theory and practice of CBPR/CPPR [6,8-10,27-30]. Despite these prior activities, sustaining the work going forward is always challenging.

\section{Creation of a shared vision}

Through the process described above, the team developed a shared vision of the HCNI and a set of goals for the CAP, specifically, to work with the community to improve health and health care in the context of a multifaceted intervention that also includes housing, employment, education and safety. The goals were to understand the health needs, health-related behaviors, health care use and clinical outcomes of residents living within a defined area of South LA.

\section{Procedures}

A focused discussion group with HCNI core members was convened in June 2013 at HAAF offices in South LA to summarize the early phases of the HCNI project. A HCNI core member is defined as a person who has been involved in HCNI since its origination in 2005. A semi-structured set of questions guided the discussion including 


\section{CAP Key Elements and Examples of Key Activities from HCNI Partnership Incorporating Key Elements}

1. Develop a memorandum of understanding to define roles of the partnership around issues such as process/procedures, perspective and time parameters. (Example: Developed a comprehensive Memorandum of Understanding (MOU))

2. Do not enter into partnership with assumptions. (Example: Articulated organizational/personal goals and time frames for each partner)

3. Value community "resident experts." (Example: Promote community members to lead/co-lead manuscripts and grant proposals; co-teach in an academic setting)

4. Establish community advisory boards, but recognize while they are important they are not sufficient for true CAPs. (Example: A Health Collaborative was established to provide feedback and insight from various community organizations with expertise in health, education, safety, employment and housing)

5. Understand how to collaborate and build effective relationships: commitment = time, patience, physical presence and assistance in building the communities' capacity for understanding, participating in and benefiting from research. (Example: Identified and committed time and resources to goals of each partner, e.g., Supported CBO activities that addressed the needs of the communities they served such as health fairs, community presentations; joint podium and poster presentations at academic meetings; support for and collaborations on academic and community grant applications; jointly developed projects, such as training guides, resource manuals, surveys, worksheets, reports for community)

6. Faculty need to be briefed/educated by community leaders and vice versa. (Example: Invited leadership of academic center to community meetings; invited leadership of community to key academic meetings; updated academic leadership on the activities of the collaborative)

7. Recognize the existence of competing agendas - be open, respectful. (Example: Clear discussions about goals and priorities of participants and their organizations; all joint documents and materials circulated to team members; developed website to ensure availability of documents)

8. Respect community's time, effort, insights (recognized with payment for services/\%effort, authorship, etc., - same as academia). (Example: In-kind contributions of time from the $\mathrm{CBO}$ and academic leaders and staff members; space provided in-kind by community partners and academic partners; hired academic staff who could facilitate meetings and work collaboratively with $\mathrm{CBO}$ staff; funding to support $\mathrm{CBO}$ s and academic partners)

9. Build on existing community resources. (Example: Trainings on depression awareness, community safety, Cardiopulmonary resuscitation (CPR), and biomarker data collection)

10. Funding source should be committed to maintaining close contact throughout the project. (Example: Awarded funds for HCNI through UCLA CTSI whom also provide academic staff support that work collaboratively with CBO staff)

11. For meetings, alternate sites, establish ground rules, maintain community and academic co-chairs (consider two community co-chairs to one academic co-chair if needed to balance power dynamics). (Example: Regular meetings - mostly in the community; initially in person, subsequently alternated face-to-face meetings with telephone calls; agendas for subsequent meeting discussed at the end of each meeting and circulated several days prior to the meeting; occasionally requested in person meetings to address important or challenging issues (e.g., to modify protocols or develop responses to IRB queries) or culmination of an internship or to celebrate important milestones (e.g., completion of data collection)

12. Mentorship: Use a model where community members are co-mentors for entry-level academic faculty who are learning skills to conduct respectful partnered research with and in communities with balanced input from both academic and community sides of the research program. (Example: Identified "trainees" from the community and from the academic sector; included undergraduate and graduate students as interns; mentored non-junior faculty members new to community-partnered research methods)

Table 1: Utilization of the Key Themes of Community-Academic Partnership (CAP) within the HCNI Partnership.

topics such as the history of HCNI, the partnership's successes and challenges, and strategies used within the partnership to overcome barriers and successfully sustain a community-academic research partnership. These topic areas are aligned with critical realism concepts to better understand how the HCNI partnership participatory research methods has added value to the research process [2,31]. The discussion was approximately two-hours and digitally recorded. The recording was de-identified and transcribed by an outside firm and reviewed by an HCNI co-investigator, independent from the discussion group process, to create themes. A trained facilitator took notes during the discussion. These notes formed the basis of three subsequent two-hour focused discussion groups with all current HCNI study team members, including core members who participated in the first group discussion. The trained facilitator took notes at these subsequent series of team meetings which distilled lessons learned in building and sustaining the HCNI CAP and using grounded theory developed a set of recommendations around forming and sustaining a CAP as previously described $[8,10,32]$. To account for group dynamics and allow differential expression of opinion, one-on-one, semi-structured, 30-minute interviews were conducted with three randomly selected HCNI core members that participated in the initial focused discussion group. The interviews were held at each member's respective office by a trained interviewer who also took notes. All interviews were digitally recorded. Members of the HCNI team (KMK, KN, AB, LJ, DM and AW) analyzed the core members' one-on-one transcripts and larger group summary notes for emerging themes and lessons learned from the partnership [32]. These analyses identified the themes that helped to form the HCNI, approaches to sustaining its development and growth and strategies for mitigating challenges. The HCNI partnership then used these themes to develop a framework for forming and sustaining CAPs to improve health and reduce disparities.

\section{Results}

The focused discussion group and subsequent review by the larger study team highlighted the key elements used to guide the HCNI partnership. The members of the HCNI team strongly endorsed the importance of CAP principles as a framework for initiating and expanding the collaborative. Table 1 lists the CAP Key Themes and provides examples of strategies consistent with each key theme that was used in HCNI to promote the partnership.

The discussants and reviewers cited several important characteristics of the project partners and the partnership itself that were essential to building and sustaining the HCNI collaborative (Table 2), including mutual respect, consistent and committed leadership, and a shared vision. Characteristics of the partners that the discussants identified as instrumental to the partnerships success included consistent and committed leadership with a shared vision and the fact that many of the original members continued to partner. Even when there was turnover at an organization, having representatives from the institution or agency who understood the project and could take on a leadership role ensured continuity. There was transparency about the needs of each member of the partnership. Each member of the partnership was asked to be explicit about his or her individual goals and the goals/needs of the agency or institution represented. At each step, the group tried to emphasize and prioritize the needs of the community. There was also an effort to ensure that the core missions of the community organizations were not undermined by participating in the project. At the same time, there was sensitivity to salient outcomes for academic partners, among them funding, 
Citation: King, KM, Morris, D, Jones, L, Lucas-Wright, A, Jones, F et al. (2015) The Los Angeles Healthy Community Neighborhood Initiative: A Ten Year Experience in Building and Sustaining a Successful Community-Academic Partnership. J Community Med Public Health Care 2: 007.

\begin{tabular}{|c|c|}
\hline Strengths & Partnership Accomplishments \\
\hline \multicolumn{2}{|l|}{ Characteristics of the Partners } \\
\hline Consistent and committed leadership with shared vision & $\begin{array}{l}\text { - Kept the study team focused on the needs of the community } \\
\text { Helped community partners and residents navigate through some of the } \\
\text { challenges of research from a community perspective. }\end{array}$ \\
\hline $\begin{array}{l}\text { Many of the original team members continue to partner, and there are generally several } \\
\text { representatives from each agency/institution involved in the project }\end{array}$ & $\begin{array}{l}\text { - Maintained continuity } \\
\text { - Presented a consistent face to the community } \\
\text { destabilize the partnership }\end{array}$ \\
\hline Transparency about needs of the community & $\begin{array}{l}\text { - Kept the needs of the community foremost } \\
\text { Used the strengths of the community to enhance partnership, study design, and } \\
\text { data collection. } \\
\text { - Pursued extramural funding for future opportunities }\end{array}$ \\
\hline Transparency about needs of academic partners & $\begin{array}{l}\text { - } \quad \text { Addressed the needs of the academic partners } \\
\text { - } \quad \text { Pursued extramural funding for future opportunities } \\
\text { Supported publications and other deliverables for academic promotion }\end{array}$ \\
\hline Transparency about the partnership & $\begin{array}{l}\text { Maintained a high level of communication and copied team members who were } \\
\text { not present so they do not feel excluded } \\
\text { Agreed that information on working group activities would be shared with the } \\
\text { larger group at a given periodicity } \\
\text { Minimized small group conversations that can be destructive and bring to the } \\
\text { entire group as soon as possible }\end{array}$ \\
\hline $\begin{array}{l}\text { Several individuals (both community and academic partners) had prior experience with } \\
\text { (CBPR/CPPR*) }\end{array}$ & $\begin{array}{l}\text { - Created a more efficient process } \\
\text { - New or less experienced members mentored by community and academic } \\
\text { members with more experience in CBPR/CPPR }\end{array}$ \\
\hline \multicolumn{2}{|l|}{ Characteristics of the Partnership } \\
\hline Trust within partnership & $\begin{array}{l}\text { Pre-existing partnerships grounded in trust helped study team work together } \\
\text { with similar assumptions, which provided a foundation for a more committed } \\
\text { partnership; } \\
\text { Helped to navigate with balance between trust and skepticism }\end{array}$ \\
\hline $\begin{array}{l}\text { Peer Governance and Egalitarian Processes that were informed by group discussions, } \\
\text { presentations by "resident" experts from community and academia, and invited } \\
\text { presentations from other individuals when outside expertise was needed }\end{array}$ & $\begin{array}{l}\text { - Improved operations and reduced tensions and hierarchies } \\
\text { - Allowed team to make informed decisions regarding study design, protocols, } \\
\text { data collection, and ethical issues within the study team }\end{array}$ \\
\hline Built trust within the community & $\begin{array}{l}\text { Strengthened community support of the project, enhanced participation of } \\
\text { stakeholders, and facilitated honest feedback and engagement of community } \\
\text { members }\end{array}$ \\
\hline Emphasis on a non-deficit, asset based approach to working with community & $\begin{array}{l}\text { Demonstrated respect and regard for community being served } \\
\text { Synthesized information on community resources that could be shared with } \\
\text { residents, local agencies, and academic institutions }\end{array}$ \\
\hline Commitment to ethical behavior within the partnership & $\begin{array}{l}\text { - Developed an expectation of fair, equitable, and respectful treatment of and by all } \\
\text { members of the partnership }\end{array}$ \\
\hline Identified benchmarks and celebrated interim successes & $\begin{array}{l}\text { Enhanced the engagement of individual participants in the process } \\
\text { - Highlighted achievements of individuals, organizations, and the HCNI team }\end{array}$ \\
\hline In-kind funding from CAP despite initial limited funding & 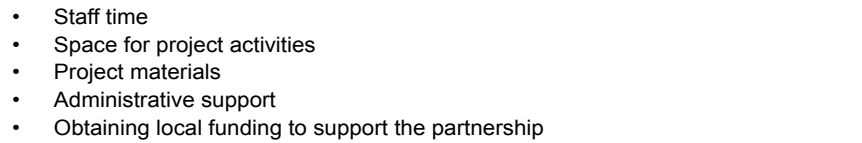 \\
\hline Recognition of mutual benefits & $\begin{array}{l}\text { Recognized these needs also had relevance for community members whose } \\
\text { employment prospects could be enhanced }\end{array}$ \\
\hline
\end{tabular}

Table 2: Factors Identified by the HCNI Team Members that Contributed to a Successful Community-Academic Partnership (CAP).

${ }^{*}$ CBPR/CPPR $=$ Community-Based Participatory Research/Community-Partnered Participatory Research, a collaborative approach to research that equitably involves all partners in the research process and recognizes the unique strengths that each brings, to jointly explore answers to complex socio-medical questions. An emphasis on partnering with communities rather than possessing a location within the community to conduct research.

publications and presentations, and academic promotion. Finally, because many of the community and academic partners had prior experience with CBPR and/or CPPR, the process of engagement was more efficient, and those with more experience were able to mentor less experienced colleagues.

The focused discussion group further identified five themes, which were related to the success of building and sustaining the HCNI partnership. These five themes were recognized as the "glue" to sustaining the CAP. Some of these themes were also included in the HCNI memorandum of understanding (MOU), a contract defining the CAP for the project.

\section{Building trust}

Trust was a critical element of the partnership's success. Several factors promoted trust within the partnership: experience participating in CAPs as described above, a long history of leadership and service within the community, and pre-existing relationships with mutual respect between the partners.

"I think another one of the strengths that assisted this project is the longstanding partnerships that had worked on many other projects together and committed and everybody that was here had a certain set of ethics that they are familiar with and work with...it was the trust that they have amongst themselves and the commitment that they had that helped it [HCNI] to foster and move forward." - Academic Partner 
A central component to building community trust was developing and maintaining community awareness of, and interest in, the project. It was also essential to leverage existing resources from each of the partners in efforts to build trust within the community. The team emphasized an asset-based approach to working with the community that involved identifying and leveraging resources from a range of community stakeholders to support the HCNI.

"...one of the things that kept going on the agenda, that we kept going back to is making sure that the instrument [survey] was culturally appropriate...we brought in a cross-section of the community and sort of ran things by them for them to take a look at and got some interesting and salient points along the way, which I think was a good thing to do so we don't get so far down the road and then find out from the community that this was a mess." - Community Partner

"But I think one of the things that we really enjoyed... was the fact that if we got it right and we got into the community and did it right, that we would have people on board with us that by word of mouth and by our behavior in community, that that would move this project forward and actually get it out there and let people see it... when we talked about this project, people really receive it with open arms." - Community Partner

\section{Transparency}

There was transparency about the needs of each member of the partnership, and at each step, the group tried to ensure that the core missions of the community organizations were not undermined by their participation. This transparency required each partnership member to share their goals as they aligned with the project. When the goals did not align, transparency was achieved through honest discussions of how new goals not previously endorsed by the project could be aligned with the project. When goals did not align with the project, transparency was maintained with discussions highlighting the importance of the shared project goals while remembering that lack of endorsement of a partner's new goals were not intended as personal slights. The partnership thrived when individuals were encouraged to bring new ideas to the group for discussion even when there was no assurance they would be accepted as new goals.

"...what the goal was there was this large project that [CP1] described and that was an opportunity for people to come in and contribute... however, a lot of people came in and saw this as their opportunity. Came in with the perspective that my independent project desires, wishes, and needs are of primary importance...I want to drive the whole project around me and my project. But it has to be, everybody has to put their personal agendas as secondary to the primary project and primary goal... those people eventually dropped off" - Community Partner

“...we had to look saying who's really working on the bus and who needs to be off the bus? So that's when we had to have meetings with [AP5] and [AP6], the person that we had to get off [the bus] because it just wasn't working. It was really bringing the morale of the rest of the group down every time we met because there was always an issue." - Community Partner

\section{Equity and fairness}

A critical element in creating equity and fairness in the partnership was through shared leadership. The partners recognized the diversity within the CAP by identifying one or more rotating leads for different components of the project, who would put forth a plan and bring it back to the rest of the group. This process was iterative and elicited an ongoing, layering discussion that helped the larger group reach decisions on project related issues. The team emphasized egalitarian processes and peer governance, which fostered a willingness and openness to questioning each other and discussing competing priorities-a process the partners described as maintaining a "balance between trust and skepticism" [33].

"For the local community that's a big piece because we as community, we're living the experience in the community. We know what the needs are but in order to develop something at the level that we needed to, we needed that academic side and [AP1], see that vision, was able to be the voice that we needed to navigate us through some of the challenges that we faced in terms of getting the expertise that we needed to be on board..." - Community Partner

"I was saying too, if you came in and sat down at the table, you couldn't tell if there was somebody leading. It was an egalitarian process. And so community and the academic side, it was equal governance, people participation across the board." - Community Partner

\section{Need for adequate resources}

The partnership was started with no formal funding; however, the partners collaborated to achieve short term projects within the community, often linking to ongoing activities their agencies or institutions, such as health fairs, local presentations on health and well-being, and community-wide conferences. The team used these events to disseminate information on the collaborative, gauge community interest in the project, and identify community priorities for the collaborative. These efforts were also central to building trust within the community by developing and maintain community awareness of an interest in the project. The team also emphasized a non-deficit, asset-based approach to working with the community that involved identifying and leveraging community resources to support the HCNI.

"That was another thing. There was no money. Everybody was working for four years with no money. So everybody, we all came to the table volunteering but we'd known in the long range we would find money or continue to look for money... it wasn't till a couple years ago that we got the CTSI funding to actually fund the project." - Community Partner

"...it was a lot of time and energy that was not, that we had not funding for, but everybody was really committed to it. So it was a challenge to make sure that we could continue doing the work without any funding to get it done." - Academic Partner

\section{Developing protocols to provide structure}

A final theme important to building and sustaining the CAP was the development of protocols, which provided structure to the process for achieving study goals and outcomes. For instance, the team developed protocols for sharing information, completing and distributing minutes, deciding on the next meeting's agenda at the end of each meeting, regular emails with the agenda and documents to be reviewed beforehand, etc.

\section{Challenges and barriers to partnering}

During the focused group discussion and the subsequent review by team members, several challenges and barriers were identified. Here we highlight the most salient challenges and barriers.

The partnership consisted of individuals from diverse backgrounds who had expertise in a range of academic disciplines and community topics. Differences in opinions and in styles of communication initially discouraged some members of the study from fully 
participating in the research process and early on, caused some study team members to leave the group. The partners worked to resolve this challenge by adopting peer governance and an egalitarian approach that encouraged all participants to voice their opinions and to question each other's assumptions respectfully.

Another challenge involved conflicting goals and agendas among team members and organizations that led to disagreements between some of the partners. At times the team strayed from main goals of the collaborative and the specific projects needed to achieve these objectives. This was especially problematic early on, before the MOU was established and finalized. Key to resolving this problem was referring back to the MOU to reiterate and prioritize the shared objectives of the partnership. This conflict resolution process was generally successful in addressing differing agendas, but in one instance, a team member who could not reconcile personal goals with the partnership objectives was asked to leave the project after many attempts to re-align competing priorities.

Another major barrier encountered was a lack of funding. This was a substantial challenge, as many of the team members were working in-kind on HCNI while maintaining full workloads in their agencies. The lack of consistent funding resulted in significant delays to many components of the project. However, the group took steps to overcome this challenge by applying for extramural and intramural funding. Eventually, the study team secured intramural funding from the UCLA CTSI and from the University of Southern California (USC)-UCLA Biodemography Center to assist with data collection, partnered analyses, manuscripts, intervention development and obtaining longer term funding for interventions.

A final major barrier was the difficulty finalizing the adult survey questions. The survey was one of three parts of the adult health interview comprised of 1) the survey, 2) health screening, and 3) laboratory data collection conducted within the household. There were protracted disagreements, limited staff and resources regarding the survey focus and which questions to include slowed progress by a year. The HCNI study team brought in new study team members to view survey in light of overall goals and community feedback and developed an iterative process to revise the survey. The new study team members fielded several drafts with community and found survey was too long. This forced the team to revisit original goals from MOU to guide focus and shorten the survey.

\section{HCNI outcomes}

Despite the challenges and barriers described above, the approaches we outlined resulted in several products and accomplishments. These include: the MOU; a partnered informed consent form; an IRB approved study protocol; training materials such as a manual of procedures, field safety protocol documents for study staff, and patient result reporting protocols. The data collection elements developed jointly through the partnership included an adult survey, a manual for neighborhood observations, a community resource guide, and mapping of community assets and deficits.

\section{Discussion}

The HCNI's CAP was founded to address health and social inequities identified in communities in South LA. We identified several key factors that helped to build the CAP and to sustain the relationships between the partners over the past seven years $[9,10]$. Consistent with the findings of Eriksson et al., [17] mutual respect and a shared vision led to shared goals and built a strong foundation for the partnership. The HCNI team built upon this foundation by incorporating five key themes for building and sustaining the partnership: trust, transparency, equity and fairness, need for adequate resources and importance of developing protocols. These mirrored the four themes of accepting different levels of participation in different phases, openly discuss mutual expectations, unmasking power and authority and allow the work to take the necessary time reported by Fröding and colleagues for using a CBPR approach to increase participation of community-academic partnerships to improve health and well-being in poor neighborhoods [16]. Lastly, we identified challenges and barriers to building and sustaining a partnership.

Here we share some of our lessons learned from building and sustaining HCNI that may be useful to other community organizations and academic institutions interested in forming or nurturing partnerships.

\section{Lessons learned}

The first lesson was to carefully think through the members of the team. It is important to frequently review who is not at the table and who should be at the table $[28,34,35]$. Importantly, this should include not only key stakeholders who might be supportive but those who might be naysayers in order to have a spectrum of perspectives and insights that allow the team to think through the potential successes and pitfalls of the project and planned strategies. While recognizing different individuals will have different agendas, we strive to have each person leave their personal agendas outside the door when they work as part of the team. Having some community and academic partners with prior experience in CAPs promoted efficiency in the partnering process, as those with more experience were able to mentor less experienced colleagues. Within HCNI, this process helped build trust among the partners. In addition, leveraging each partner's existing resources helped build trust in the project with the community.

Aligning individual/organization-specific goals and the group agenda through a project-specific MOU helped to reinforce and prioritize the shared objectives of the partnership. This process helped to resolve conflicts between partner organizations. Transparency among the partners helped to promote sensitivity to salient outcomes for each partner, such as publications, presentations, and academic promotion for academic partners and capacity needs for community partners. Resonant with the findings of Fröding et al., [16] there were concerns that power differentials between individuals at the table might impede discussions about issues within the partnership and an additional concern that different communication styles might contribute to separate discussions among partner members who did not feel comfortable approaching some members of the group. To address these concerns, the partners developed a shared leadership and peer governance structure that encouraged all participants to voice their opinions and to question each other's assumptions respectfully in a manner similar to that described by Jones et al. [36]. This process was described by the partners as "maintaining a balance between trust and skepticism" [33] that contributed to timely and efficient conflict resolution. In addition, the senior community and academic partners having a long history of collaboration further facilitated power sharing.

Another challenge was maintaining project continuity. Over time multiple transitions occur, some are temporary and some permanent. When there was turnover of HCNI representation at an organization, it was important for incumbent partners to connect the new team 
member to the primary goals of the project to ensure continuity as well as to recognize people may have to leave and may also rejoin during the course of the project [37].

Lack of resources was one of the biggest challenges in sustaining the partnership. In some instances, trying to achieve the mission of the respective partners while staying committed to HCNI significantly decreased productivity towards the study aims. However, the group took steps to overcome this challenge by appointing a working group within the partnership that consisted of community and academic team member who focused on obtaining extramural and intramural funding. The study team secured intramural funding from the UCLA CTSI and from the USC-UCLA Biodemography Center as noted earlier.

Lastly, developing protocols provided structure for the partners and the project. By having specific protocols in place for sharing information, the conduct of meetings, etc., the CAP was able to work more efficiently to achieve project goals and outcomes and to celebrate small successes at each phase of the project. A unique but related challenge that emerged from the individual interviews with core HCNI members was the Institutional Review Board challenges for study approval. CAP projects such as HCNI revealed distinctive IRB requirements leading to multiple revisions to the study protocol before it was approved. After approval, the project coordinator continued close communication with IRB to ensure all requirements were being met each approval period.

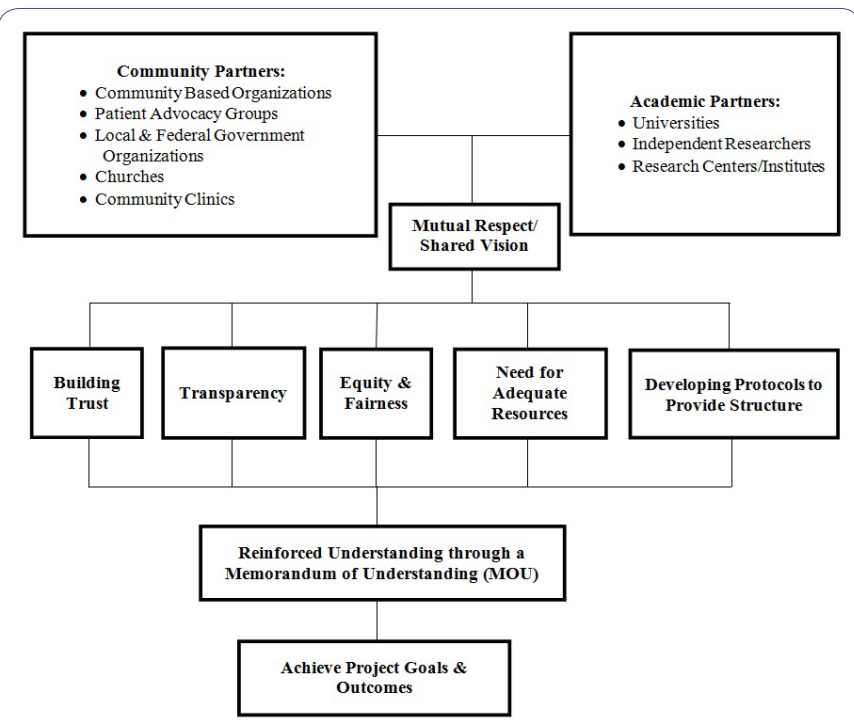

Figure 1: Community-Academic Partnership Framework.

Based on our discussions, we developed a Community-Academic Partnership Framework (Figure 1) that includes the core themes that we believe are essential to building and sustaining the partnership. These elements should be reinforced through the MOU and thus guide the partners in sustaining and strengthening their relationships and remaining aligned with the project goals and outcomes.

\section{Conclusion}

Building a CAP to improve community health is not an easy feat. It takes time, patience, and commitment to the process. HCNI was successful in building and sustaining a partnership that achieved some of the initial study goals, but it also benefited from several years of prior collaborations. The next steps for the HCNI are to discuss the challenges and successes of the initial study goals, analyze the data collected, and use the data, in partnership with the community, to develop an intervention that will address the HCNI vision to impact policy for improving community health $[38,39]$.

\section{Acknowledgements}

The project described was supported in part by the National Center for Advancing Translational Sciences through UCLA CTSI Grant UL1TR000124 and the USC-UCLA Center of Biodemography \# 5P30AG017265-13. The content is solely the responsibility of the authors and does not necessarily represent the official views of the $\mathrm{NIH}$. The funder was not involved with the design and conduct of the study; collection, management, analysis and interpretation of the data; and preparation, review, or approval of the manuscript.

The HCNI study group includes Healthy African American Families:Dennishia Banner, Felica Jones, Karina Madrigal, BA, Gabriela Gomez, BA, Nancy Hernandez, Tori Bailey; Los Angeles Urban League: Astrea Flowers, MSW, Linda Thompson, MSW; Charles R. Drew University: Nell Forge, PhD, Laurice Pitts, LVN, Dale McNeal, Kesha Cargill; University of California, Los Angeles: Ibrahima Sankare, MHA, Juan Barron, BA, Blanca Corea, MA, Arturo Martinez, BA, Stefanie D. Vassar, MS, Lujia Zhang, BS; LA Biomed/Harbor UCLA: Rachelle Bross, PhD.

We also want to thank the LAUL Health Collaborative, HCNI participants and the residents of the South Los Angeles community for their support.

\section{References}

1. Petersen R, Hunkins JA, Riegel LD, Smith L (2012) Forging new partnerships to build healthier communities for a healthier state. N C Med J 73: 270-273.

2. Jagosh J, Macaulay AC, Pluye P, Salsberg J, Bush PL, et al. (2012) Uncovering the benefits of participatory research: implications of a realist review for health research and practice. Milbank Q 90: 311-346.

3. Israel BA, Schulz AJ, Parker EA, Becker AB (1998) Review of community-based research: assessing partnership approaches to improve public health. Annu Rev Public Health 19: 173-202.

4. Wallerstein NB, Duran B (2006) Using community-based participatory research to address health disparities. Health Promot Pract 7: 312-323.

5. Israel BA, Schulz AJ, Parker EA, Becker AB, Community-Campus Partnerships for Health (2001) Community-based participatory research: policy recommendations for promoting a partnership approach in health research. Educ Health (Abingdon) 14: 182-197.

6. Ferre CD, Jones L, Norris KC, Rowley DL (2010) The Healthy African American Families (HAAF) project: from community-based participatory research to community-partnered participatory research. Ethn Dis. Winter 20: 2-1-8.

7. Jones $L$ (2009) Preface: Community-partnered participatory research: how we can work together to improve community health. Ethn Dis 19: 1-2.

8. Jones L, Wells K (2007) Strategies for academic and clinician engagement in community-participatory partnered research. JAMA 297: 407-410.

9. Wells KB, Staunton A, Norris KC, Bluthenthal R, Chung B, et al. (2006) Building an academic-community partnered network for clinical services research: the Community Health Improvement Collaborative (CHIC). Ethn Dis. Winter 16: 3-17.

10. Norris KC, Brusuelas R, Jones L, Miranda J, Duru OK, et al. (2007) Partnering with community-based organizations: an academic institution's evolving perspective. Ethn Dis 17: 27-32.

11. Jones L, Wells K, Meade B, Forge NG, Aziza Lucas-Wright, et al. (2009) Work through the valley: Do. Ethnicity \& disease 19: 6-39-46. 
12. Minkler M, Wallerstein N (2008) Community-based participatory research for health: From process to outcomes. ( $2^{\text {nd }}$ edn). Wiley, New York, USA.

13. Aguilar-Gaxiola S, Ahmed S, Franco Z, Kissack A, Gabriel D, et al. (2014) Towards a unified taxonomy of health indicators: academic health centers and communities working together to improve population health. Acad Med 89: $564-72$.

14. Wallerstein N, Duran B (2010) Community-based participatory research contributions to intervention research: the intersection of science and practice to improve health equity. Am J Public Health. 100: 40-46.

15. Israel BA, Schulz AJ, Park ER, Becker AB, Allen AJ et al. (2003) Critical issues in developing and following community based participatory research principles. In: Minkler M, Wallerstein N (eds.). Community Based Participatory Research for Health. Jossey-Bass Publishers, San Francisco, USA.

16. Froding K, Elander I, Eriksson C (2014) A community-based participatory research process in a poor Swedish neighbourhood. Systemic Practice and Action Research 28: 19-36.

17. Eriksson CC, Fredriksson I, Froding K, Geidne S, Pettersson C (2014) Academic practice-policy partnerships for health promotion research: experiences from three research programs. Scand J Public Health 42: 88-95.

18. Wong E, Chung B, Stover G, Stockdale S, Jones F, et al. (2011) Addressing unmet mental health and substance abuse needs: A partnered planning effort between grassroots community agencies, faith-based organizations, service providers, and academic institutions. Ethn Dis 21: 1-107-113.

19. Friedman D, Johnson KM, Owens OL, Thomas TL, Dawkins DS, et al. (2012) Developing partnerships and recruiting dyads for a prostate cancer informed decision making program: Lessons learned from a community-academic-clinical team. J Cancer Educ 27: 243-249.

20. Phillips-Angeles E, Song L, Hannon PA, Celedonia M, Stearns S, et al. (2013) Fostering partnerships and program success. Cancer 119: 2884-2893.

21. Chandra A, Williams M, Plough A, Stayton A, Wells KB, et al. (2013) Getting actionable about community resilience: The Los Angeles County community disaster resilience project. Am J Public Health 103: 1181-1188.

22. Sword W, Niccols A, Yousefi-Nooraie R, Dobbins M, Lipman E, et al. (2013) Partnerships Among Canadian Agencies Serving Women with Substance Abuse Issues and Their Children. Int J Ment Health Addict 11: 344-357.

23. Maclntyre LM, Waters CM, Rankin SH, Schell E, Laviwa J, et al. (2013) How community trust was gained by an NGO in Malawi, Central Africa, to mitigate the impact of HIVIAIDS. J Transcult Nurs 24: 263-270.

24. Ndulue U, Perea FC, Kayou B, Sprague-Martinez L (2012) Team-building activities as strategies for improving community-university partnerships: Lessons learned from Nuestro Futuro Saludable. Prog Community Health Partnersh 6: 213-218.
25. The State of Black Los Angeles (2005) Los Angeles, CA.

26. Ferré CD, Jones L, Norris KC, Rowley DL (2010) The Healthy African American Families (HAAF) project: from community-based participatory research to community-partnered participatory research. Ethn Dis 20: 2-1-8.

27. Wells K, Jones L (2009) "Research" in community-partnered, participatory research. JAMA 302: 320-321.

28. Jones L, Meade B, Norris K, Lucas-Wright A, Jones F, et al. (2009) Develop a vision. Ethn Dis 9: 6-17-30.

29. Wells KB, Springgate BF, Lizaola E, Jones F, Plough A (2013) Community engagement in disaster preparedness and recovery: a tale of two cities--Los Angeles and New Orleans. Psychiatr Clin North Am 36: 451-466.

30. Miranda J, Ong MK, Jones L, Chung B, Dixon EL, et al. (2013) Community-partnered evaluation of depression services for clients of community-based agencies in under-resourced communities in Los Angeles. J Gen Intern Med 28: 1279-1287

31. Jagosh J, Pluye P, Macaulay AC, Salsberg J, Henderson J, et al. (2011) Assessing the outcomes of participatory research: protocol for identifying, selecting, appraising and synthesizing the literature for realist review. Implement Sci 6: 24.

32. Calderón JL, Baker RS, Fabrega H, Conde JG, Hays RD, et al. (2006) An ethno-medical perspective on research participation: a qualitative pilot study. MedGenMed 8: 23.

33. Jones L, Wells K, Norris K, Meade B, Koegel P (2009) The vision, valley, and victory of community engagement. Ethn Dis 19: 6-3-7.

34. Cargo M, Mercer SL (2008) The value and challenges of participatory research: strengthening its practice. Annu Rev Public Health 29: 325-350.

35. Fröding K, Elander I, Eriksson C (2012) Neighbourhood development and public health initiatives: who participates? Health Promot Int 27: 102-116.

36. Jones D, Franklin C, Butler BT, Williams P, Wells KB, et al. (2006) The Building Wellness project: a case history of partnership, power sharing, and compromise. Ethn Dis 16: 54-66.

37. Bluthenthal RN, Jones L, Fackler-Lowrie N, Ellison M, Booker T, et al. (2006) Witness for Wellness: preliminary findings from a community-academic participatory research mental health initiative. Ethn Dis 16: 18-34.

38. Green LW, Glasgow RE, Atkins D, Stange K (2009) Making evidence from research more relevant, useful, and actionable in policy, program planning, and practice slips "twixt cup and lip". Am J Prev Med 37: 187-191.

39. Green LW (2014) Closing the chasm between research and practice: evidence of and for change. Health promotion journal of Australia: official journal of Australian Association of Health Promotion Professionals. 25: 25-29. 